\title{
Protection against Taenia pisiformis larval infection induced by a recombinant oncosphere antigen vaccine
}

\author{
L. Chen ${ }^{1 *}$, D.Y. Yang ${ }^{1 *}$, Y. Xie ${ }^{1}$, X. Nong ${ }^{1}$, X. Huang ${ }^{1}$, Y. Fu ${ }^{1}$, X.B. Gu ${ }^{1}$, \\ S.X. Wang ${ }^{1}$, X.R. Peng ${ }^{2}$ and G.Y. Yang ${ }^{1}$ \\ ${ }^{1}$ Department of Parasitology, College of Veterinary Medicine, \\ Sichuan Agricultural University, Ya'an, China \\ ${ }^{2}$ College of Life and Basic Science, Sichuan Agricultural University, Ya'an, China \\ *These authors contributed equally to this study. \\ Corresponding author: G.Y. Yang \\ E-mail: guangyou1963@aliyun.com
}

Genet. Mol. Res. 13 (3): 6148-6159 (2014)

Received September 5, 2013

Accepted December 12, 2013

Published February 13, 2014

DOI http://dx.doi.org/10.4238/2014.February.13.14

\begin{abstract}
Taenia pisiformis larvae cause significant health problems to rabbits. At present, it is not known whether the recombinant antigen from the $T$. pisiformis oncosphere is able to confer protective immunity against T. pisiformis larval infection. The full-length cDNA was cloned into a pET32a $(+)$ vector, and the recombinant protein was then expressed in BL21 (DE3) cells. Vaccination with the purified $r \mathrm{TpUbc} 2$ coupled with QuilA was carried out in New Zealand rabbits to evaluate the immunoprotective effect against $T$. pisiformis infection. The full-length open reading frame of the TpUbc2 gene was 444 bp, and encoded a 16.63-kDa protein. Finally, $r \mathrm{TpUbc} 2$ was used to evaluate the ability to induce immunoprotective responses in rabbits. A 79.3-90.8\% reduction $(\mathrm{P}<0.01)$ in the recovery of larvae was observed in the experimental group compared to the control group. Specific anti-rTpUbc2 antibodies from immunized rabbits had significantly higher levels of $\operatorname{IgG}(\mathrm{P}<0.01)$ compared to the control group; however, no significant difference in IgA levels was found between groups
\end{abstract}


( $\mathrm{P}>0.05)$. Our data support the use of $r \mathrm{TpUbc} 2$ as a potential candidate to develop a vaccine against $T$. pisiformis larvae.

Key words: Taenia pisiformis; Ubiquitin-conjugating enzyme; Immunization; Vaccine

\section{INTRODUCTION}

The adult stage of Taenia pisiformis (Cestoidea; Cyclophyllidea; Taeniidae) parasitizes the small intestine of canines and felines (Saeed et al., 2006; Lahmar et al., 2008; Bagrade et al., 2009; Jia et al., 2010). T. pisiformis larvae infect the liver capsule, greater omentum, and mesentery of rabbits, which serve as an intermediate host. T. pisiformis is widely distributed across the world (Foronda et al., 2003; Martinez-Moreno et al., 2007; Zhou et al., 2008). Infections may occur when canines ingest the internal organs of rodents infected with T. pisiformis larvae, or when lagomorphs consume food contaminated with the proglottids of T. pisiformis. T. pisiformis causes significant health problems to its intermediate and definitive hosts (Rajasekariah et al., 1985).

Recombinant antigen has been proposed as candidates for a future vaccine against a variety of helminth parasites. Since the early 1990s, more than 80 different recombinant antigens from 22 different helminth species have been studied (Geldhof et al., 2007). Effective recombinant vaccines have been developed for cestode parasites, including Taenia ovis, $T$. solium, T. saginata, and Echinococcus granulosus (Lightowlers, 2006).These studies provide important information that could be used for the development of a genetically engineered vaccine against $T$. pisiformis larvae. Previous studies on T. pisiformis have mainly focused on observing its morphology (Shield et al., 1973), biological characteristics (Kyngdon et al., 2006; Zhou et al., 2008; Toral-Bastida et al., 2011), epidemiology (Rashed et al., 1991), and treatment (Rajasekariah et al., 1985). To date, very little is known about recombinant antigens conferring protective immunity against $T$. pisiformis infection.

The ubiquitin-dependent proteolytic system is essential for the maintenance of homeostasis in a broad range of eukaryotic proteins. It is a major pathway for protein degradation in eukaryotes, and plays a vital role in the control of numerous cellular processes (Finley et al., 1989). Ubiquitin is conjugated to the target protein via a cascade of enzymatic reactions, which involve an ubiquitin-activating enzyme (E1), an ubiquitin-conjugating enzyme (E2 or UBC2), and an ubiquitin-protein ligase (E3). E2 enzymes act via selective protein-protein interactions with the E1 and E3 enzymes and, in doing so, lead to different effects on downstream substrates that have either a single or chain of $\mathrm{Ub} / \mathrm{UBL}$ molecules. In addition, E2 has recently proven to be a key mediator within the ubiquitin chain assembly (Ye and Rape, 2009).

In this study, we isolated the ubiquitin-conjugating enzyme gene of the T. pisiformis oncosphere based on the analysis of expressed sequence tags (Yang et al., 2012). The recombinant protein $(r \mathrm{TpUbc} 2)$ was expressed, analyzed, and purified for the immunization of rabbits.

\section{MATERIAL AND METHODS}

\section{Rabbits}

Twenty-eight, 60-day-old female white New Zealand rabbits were purchased from the 
Laboratory Animal Center of Sichuan Agricultural University (China). All animals from which specimens were collected were handled in accordance with the animal protection law of the People's Republic of China (a draft of the animal protection law in China was released on September 18, 2009). This study was approved by the National Institute of Animal Health Animal Care and Use Committee at Sichuan Agricultural University (approval number 2010-021).

\section{Parasite eggs}

Eggs of T. pisiformis, which originated from 5 different worms, were collected from 1 experimentally infected dog in the Department of Parasitology, College of Veterinary Medicine, Sichuan Agricultural University (China).

\section{Total RNA isolation and amplification of TpUbc2}

Total RNA was isolated from the active oncosphere of $T$. pisiformis using an RNA isolation kit (TaKaRa, Dalian, China), and first-strand cDNA synthesis was performed using a cDNA synthesis kit (TaKaRa) and an oligo (dT) 18 primer (TaKaRa). The resulting cDNA was used for amplification by polymerase chain reaction (PCR) using a sense primer (5'-ATGGCACTCAAAAGAATCCAGAAGG-3') and an antisense primer (5'-TCACATCGCGTACTTCTGTGTCCAT-3'). PCR products were separated by electrophoresis on agarose gel (1\%), purified using a Gel Extraction Kit (TIANGEN, Beijing, China), following manufacturer protocols, and cloned into a pMD19-T vector (TaKaRa, Dalian, China), following manufacturer protocols. The resultant plasmid was transferred to Escherichia coli strain DH5 $\alpha$ (TIANGEN), and sequenced.

\section{DNA sequence analysis, B cell epitope prediction, and cross-reactivity prediction}

An Open Reading Frame (ORF) Finder (http://www.ncbi.nlm.nih.gov/projects/gorf/) and the BLAST network server of the National Center for Biotechnology Information (http:// blast.ncbi.nlm.nih.gov/Blast.cgi) were used to analyze the ORF, nucleotide sequence, and deduced amino acid sequence of $\mathrm{TpUbc2}$, to determine their similarities with previously reported sequences in the current database. Analysis of the signal sequence was performed using the SignalP 3.0 Server (http://www.cbs.dtu.dk/services/SignalP/). B cell epitopes were predicted using the web server of Immune Epitope Database Analysis (IEDA) (http://tools. immuneepitope.org/tools/bcell/iedb_input). Cross-reactivity with known allergens was predicted using the web server Structural Database of Allergenic Proteins (SDAP) (http://fermi. utmb.edu/SDAP/).

\section{Expression and purification of the recombinant TpUbc2 fusion protein}

The coding region of TpUbc2 was amplified by PCR using a sense primer (5'-CGC GGATCCATGGCACTCAAAAGAATCC-3') containing a BamHI site and an antisense primer (5'-CCCAAGCTTTCACATCGCGTACTTCTGT-3') containing a HindIII site. The PCR fragments were digested with BamHI and HindIII (TaKaRa) and ligated into the BamHI and HindIII sites of the plasmid expression vector pET32a (+) (TaKaRa). E. coli strain BL21 (DE3) 
cells were transformed with the recombinant constructs using standard methods (Sambrook, 2001). Transformant colonies were evaluated by DNA restriction analysis of the plasmid. $E$. coli strain BL21 (DE3) (TIANGEN) harboring the recombinant plasmid was propagated in lysogeny broth medium $(50 \mathrm{mg}$ ampicillin, $5 \mathrm{~g}$ Bacto yeast extract, and $10 \mathrm{~g} \mathrm{NaCl}$ per liter of distilled water, $\mathrm{pH}$ 7.0), and expression was induced with $1 \mathrm{mM}$ IPTG (isopropyl $\beta$-D-1-] thiogalactopyranoside) at $37^{\circ} \mathrm{C}$ for $3 \mathrm{~h}$. Purification of recombinant $\mathrm{TpUbc} 2$ was carried out following previously described methods (Chen et al., 2003).

\section{Immunoblot analysis}

SDS-PAGE analysis was performed using $14 \%$ polyacrylamide gels with a recombinant protein electrophoresis system. Electrophoresis was carried out at a constant voltage of $160 \mathrm{~V}$ for $180 \mathrm{~min}$. Following electrophoresis, the proteins were transferred to nitrocellulose membranes (Millipore, Dermstadt, Germany), and the membranes were blocked with $1 \%$ bovine serum albumin (BSA)-PBS-Tween $(0.05 \%$ PBST) for $2 \mathrm{~h}$ at room temperature. Serum from rabbits infected with $T$. pisiformis at a dilution of 1:500 was used to detect the presence of $r \mathrm{TpUbc} 2$. After the membranes were washed 5 times with PBST, horseradish peroxidaseconjugated goat anti-rabbit IgG (AMRESCO, TX, USA) was used as a secondary antibody at a dilution of 1:8000. After the membranes were again washed 5 times with PBST, the signal was visualized with 3,3',5,5'-tetramethyl benzidine (TMB; TIANGEN) following the manufacturer protocol.

\section{Challenge infection and sampling}

The rabbits were randomly divided into 4 groups of 7 rabbits. Specifically: 1) group A was immunized with $0.9 \%$ sodium chloride solution (NS) as the control; 2) group B was immunized with $100 \mu \mathrm{g}$ QuilA $(0.01 \mathrm{~g} / \mathrm{mL}$; International Laboratory, USA) in $0.9 \%$ sodium chloride solution, as the adjuvant control; 3) and 4) groups $\mathrm{C}$ and $\mathrm{D}$ were immunized with $100 \mu \mathrm{g}$ fresh $r \mathrm{TpUbc} 2$ (histidine fusion protein) mixed with $100 \mu \mathrm{g}$ QuilA. The antigen was reconstituted in sterile deionized water immediately before the vaccination of the rabbits. All animals were immunized twice by subcutaneous immunization at 1-week intervals. Two weeks after the 2 nd vaccination, each rabbit received an oral challenge infection with 5000 viable eggs. Blood samples were collected from auricular veins of all rabbits, and were taken 1) immediately before vaccination and then 2) once a week following the vaccination and challenge, until week 7 post-challenge. The rabbits were sacrificed 50 days after infection. All cysticerci in the peritoneal cavity (greater omentum, mesentery, liver capsule, and mesorectum) were counted. The percentage reduction was calculated as: reduction rate $(\%)=[1$ - (mean number of cysticerci with vaccination / mean number of cysticerci for NS) x 100\%].

\section{Enzyme-linked immunosorbent assay (ELISA)}

Serum samples were collected from immunized and control rabbits to assess antibody responses. Serum levels of antigen-specific IgG and IgA antibody were measured by an ELISA. The recombinant proteins were bound to 96 -well microtiter plates by incubating 2 $\mu \mathrm{L} / \mathrm{mL} r \mathrm{TpUbc} 2$ in $0.1 \mathrm{M}$ carbonate buffer $\left(10 \mathrm{mM} \mathrm{Na}_{2} \mathrm{CO}_{3}, 30 \mathrm{mM} \mathrm{NaHCO}_{3}, \mathrm{pH} 9.6\right)$ for 16 
$\mathrm{h}$ at $4{ }^{\circ} \mathrm{C}$, after which they were washed 5 times with $0.05 \%$ PBST. Wells were blocked with $100 \mu \mathrm{L}$ PBST containing 5\% BSA for $1 \mathrm{~h}$ at $37^{\circ} \mathrm{C}$. After the wells were washed 5 times with PBST, $100 \mu \mathrm{L}$ rabbit serum (diluted 1:500) was added and incubated at $37^{\circ} \mathrm{C}$ for $1 \mathrm{~h}$. Then the plates were washed 6 times in PBST, and probed with 1:10,000 peroxidase-conjugated goat anti-rabbit IgG (AMRESCO) or 1:10,000 goat anti-rabbit IgA (AMRESCO) and diluted in 1\% sodium caseinate-PBST. The plates were incubated for $1 \mathrm{~h}$ at $37^{\circ} \mathrm{C}$, followed by a further 6 washes with PBST. Then $100 \mu \mathrm{L}$ TMB substrate $(45 \mathrm{mM}$ dibasic sodium phosphate, $0.22 \mathrm{mM}$ citric acid, $0.42 \mathrm{mM} \mathrm{TMB}$, and $30 \% \mathrm{H}_{2} \mathrm{O}_{2}$; TIANGEN) was added to each well. Both the test sera and conjugates were diluted in PBS, $\mathrm{pH}$ 7.5. The reaction was stopped after $30 \mathrm{~min}$ by the addition of $100 \mu \mathrm{L} 1 \% \mathrm{SDS}$, and the optical densities were read at $450 \mathrm{~nm}$ (Dynatech MR 500 , Deckendorf, Germany). A positive control, a negative control, and a blank control were always included on each plate.

\section{Statistical analysis}

Data were processed in Excel 2010. The number of cysticerci between experimental groups was statistically compared using the non-parametric Mann-Whitney test. The two-side $P$ values $<0.05$ were considered as statistically significant. The antibody data between experimental groups were compared by analysis of variance (ANOVA), The Dunnett test, and least significant difference (LSD). Differences with $\mathrm{P}$ values $<0.05$ were considered as statistically significant. Statistical analyses were performed using SPSS version 8.0 for windows.

\section{RESULTS}

\section{TpUbc 2 cDNA cloning and structural analysis}

A cDNA sequence was obtained with an ORF of 444 bp (GenBank accession No. KC182511) to encode a protein of 147-amino acid residues. The molecular mass of the peptide was calculated as $16.63 \mathrm{kDa}$ and the isoelectric point as 6.77. No signal peptide or transmembrane domains were evident in the protein, indicating that the peptide chain is not anchored to a cellular membrane. The alignment of T. pisiformis TpUbc2 with other ubiquitin-conjugating enzyme proteins is shown in Figure 1. The amino acid sequence of TpUbc2 is rich in Pro (10.20\%), Asp (9.52\%), and Leu (8.84\%) amino acids, and highly homologous to Litopenaeus vannamei, Oreochromis niloticus, Oikopleura dioica, and Danio rerio ubiquitin-conjugating enzyme proteins with identities of $88,88,87$, and $86 \%$, respectively.

\section{B cell epitope prediction}

Identification of epitopes within proteins may be useful for diagnostic purposes and in the development of peptide vaccines. B cell epitope prediction is based on experimental antigenic determinant data and the physicochemical properties of amino acid residues. These parameters are able to predict antigenic determinants with an accuracy of approximately $75 \%$ (Kolaskar and Tongaonkar, 1990). Based on the Kolaskar and Tongaonkar antigenicity methodology, 8 possible B cell epitopes were predicted in the TpUbc2 protein in T. pisiformis (Table 1). 
Taenia pisiformis Litopenaeus vannamei Oreochromis niloticus Oikopleura dioica Danio rerio

Taenia pisiformis Litopenaeus vannamei Oreochromis niloticus Oikopleura dioica Danio rerio

Taenia pisiformis Litopenaeus vannamei oreochromis niloticus oikopleura dioica

Danio rerio
MALKR IQKE LSDL GRD PPAQCS AGPVGDDLFHWQAT IMGPSD SPF EGGVFFL DIH FPTDY MALKR INKE LQDL GRD PPA QCS AGPVGDDLFHWQAT IMGPTD SPF QGGV FFLNIH FPTDY MALKR IHKE LNDLARD PPA QCS AGPVGDDMFHWQAT IMGPSD SPY QGGVFFLTIHFPTDY MALKR INKE LQDI GRD PPAQCSAGPVGDDLFHWQAT IMGPSD SPY QGGVFFLNIH FPNDY MA LKR IHKE LHDL GRD PPA QCS AGPVGDDMFHWQAT IMGPND SPY QGGVFFLTIHFPTDY

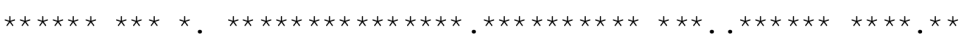

PFKPPKITFTTRI YHPNIN SNGNICLDILRNQWSPALT ISKV LLS ICSLLTD PNP DDPLS PFKPPKVAFTTRI YHP NIN SNG SICLDILRTQW SPALT ISKV LLS ICSLLTD PNP DDPLV PFKPPKVAFTTRI YHP NINSNGSICLDILRSQWSPALT ISKV LLS ICSLLCD PNP DDPLV PFKPPKVSF ITRI YHP NIN SNG SIC LDILRSQWSPALT ISKV LLS ICSLLTD PNP DDPLV PFKPPKVAFTTRI YHP NIN SNG SIC LDILRSQW SPALT ISKV LLS ICSLLCD PNP DDPLV

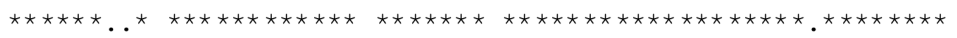

PDIARTYKTDRQKYDKTAKEWTQKYAM PE IARQYKT DREKYNK LAQEWTHKYAQ PE IAR IYKTDSQKYTKMAKEWTQKYAM PE IARVYKT DRDKYQK LAREWTQKYAM PE IAR IYKT DREKYNR IAREWTQKYAM

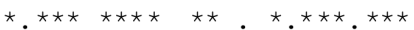

Figure 1. Comparison of TpUbc2 with the ubiquitin-conjugating enzyme E2 amino acid sequences of other species. GenBank accession numbers: Litopenaeus vannamei, ABI98679; Oreochromis niloticus, XP003442904; Oikopleura dioica, CBY19808; Danio rerio, NP957253.

Table 1. B cell epitope predictions in Taenia pisiformis TpUbc2.

\begin{tabular}{lcclrr}
\hline No. & Start position & End position & Peptide & Peptide length & Value \\
\hline 1 & 4 & 11 & KRIQKELS & 8 & 1.056 \\
2 & 18 & 25 & PAQCSAGP & 22 & 7.065 \\
3 & 46 & 67 & EGGVFFLDIHFPTDYPFKPPKI & 1.069 \\
4 & 71 & 77 & TRIYHPN & 7 & 1.132 \\
5 & 83 & 89 & NICLDIL & 1.156 \\
6 & 94 & 112 & SPALTISKVLLSICSLLTD & 1.161 \\
\hline
\end{tabular}

\section{Prediction of cross-reactivity}

A FASTA alignment was used to determine whether the TpUbc2 protein is potentially cross-reactive based on the Food and Agricultural Organization (FAO)/World Health Organization (WHO) allergenicity rules. The results showed that TpUbc2 was related to 8 allergenic proteins from 7 species (Table 2). The most homologous protein to TpUbc2 in the SDAP was found to be Asc s 1.0101 (Ascaris suum) isolated from pig ascarids $(\mathrm{E}<0.01$; Table 2).

\section{Expression of recombinant TpUbc2}

The putative TpUbc2 cDNA ORF was amplified by PCR and cloned into the expression vector pET32a $(+)$. SDS-PAGE analysis showed that the molecular mass of the expressed protein was approximately $36.63 \mathrm{kDa}$, and contained the predicted $16.63-\mathrm{kDa}$ TpUbc2 protein 
and an additional peptide expressed from the pET-32a (+) vector $(20 \mathrm{kDa})$ (Figure 2). Recombinant TpUbc2 was confirmed to be a soluble protein.

Table 2. Predicted cross-reactivity between TpUbc2 and known allergens in SDAP.

\begin{tabular}{|c|c|c|c|c|c|c|}
\hline No. & Allergen & Accession No. & Species & Sequence length & Bit score $^{a}$ & E score \\
\hline 1 & Asc s 1.0101 & AF051702 & Ascaris suum & 767 & 54.9 & $3.0 \mathrm{e}-09$ \\
\hline 2 & Ves v 6.0101 & G8IIT0 & Vespula vulgaris & 1312 & 41.2 & $4.1 \mathrm{e}-05$ \\
\hline 3 & Sola t 4 & CAA45723 & Solanum tuberosum & 165 & 38.1 & $3.4 \mathrm{e}-04$ \\
\hline 4 & Ber e 2 & AAO38859 & Bertholletia excelsa & 343 & 37.0 & $7.6 \mathrm{e}-04$ \\
\hline 5 & Fag e 1 & Q9XFM4 & Fagopyrum esculentum & 401 & 36.5 & $1.1 \mathrm{e}-03$ \\
\hline 6 & Ses i 6.0101 & Q9XHP0 & Sesamum indicum & 353 & 35.9 & $1.7 \mathrm{e}-03$ \\
\hline 7 & Sola t 4 & P30941 & Solanum tuberosum & 168 & 34.1 & $5.6 \mathrm{e}-03$ \\
\hline 8 & Sch c 1.0101 & D8Q9M3 & Schizophyllum commune & 468 & 33.6 & $7.9 \mathrm{e}-03$ \\
\hline
\end{tabular}

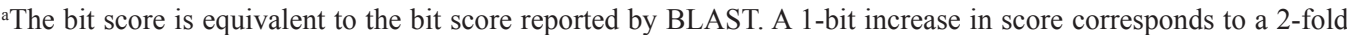
reduction in expectation, and a 10-bit increase implies 1000-fold lower expectation. Sequences with $\mathrm{E}$ values $<0.01$ are almost always homologous. SDAP $=$ Structural Database of Allergenic Proteins.

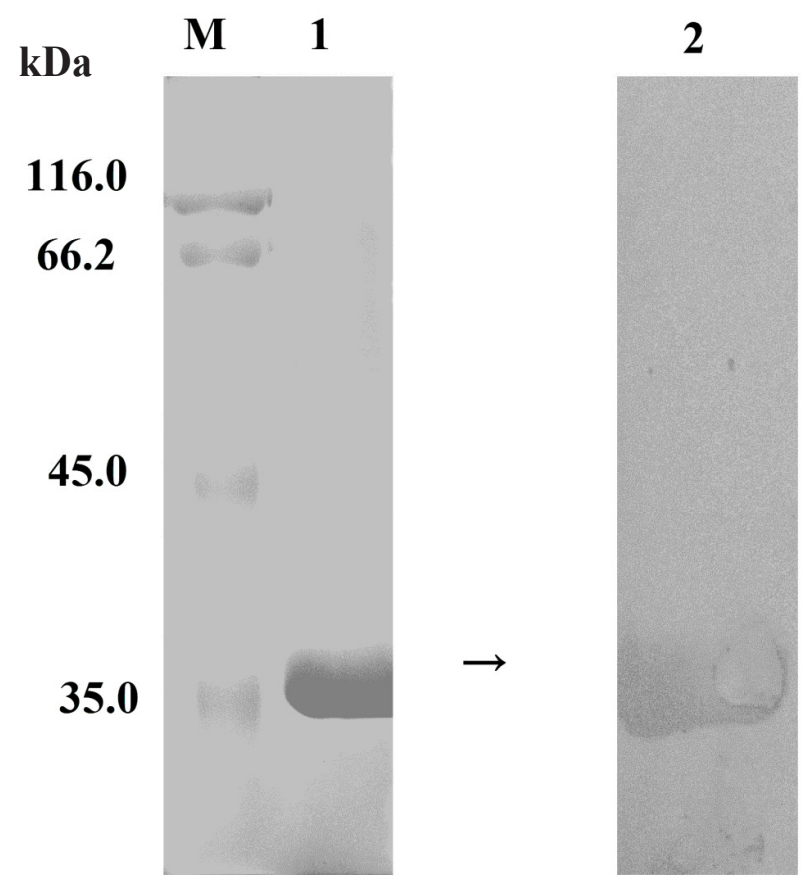

Figure 2. Western blot analyses of recombinant proteins. Lane $M=$ protein marker; lane $1=$ purified recombinant proteins; lane 2 = Western blot results.

\section{Western blot analysis}

Western blot analysis showed that the purified recombinant protein $(r \mathrm{TpUbc} 2)$ reacted with sera from rabbits inoculated with $T$. pisiformis viable eggs (Figure 2), while no reaction was observed using the negative control serum (data not shown). 


\section{Protection against the $T$. pisiformis egg challenge}

The protective effect against $T$. pisiformis larvae in rabbits inoculated with recombinant TpUbc2 was identified by counting the number of cysticerci 50 days after challenge with T. pisiformis eggs. The number of cysticerci in the vaccinated groups was reduced by $79.3 \%$ (42.6/206.0) and 90.8\% (19.0/206.0), compared with the control (NS group). Table 3 shows the numbers of peritoneal cysticerci found in the NS group, QuilA alone group, and $r \mathrm{TpUbc2}-$ QuilA immunized rabbit group.

Table 3. Reduction in the number of Taenia pisiformis cysticerci in rabbits immunized with the $r \mathrm{TpUbc} 2$ vaccine.

\begin{tabular}{lcc}
\hline Vaccine group & Mean number of cysticerci (means \pm SD) & Reduction $(\%)$ \\
\hline A (Control) & $206.0 \pm 157.0^{\mathrm{a}}$ & $108.7 \pm 137.8^{\mathrm{a}}$ \\
B (QuilA) & $42.6 \pm 28.5^{\mathrm{b}}$ & 79.3 \\
C $(r$ TpUbc+QuilA) & $19.0 \pm 13.5^{\mathrm{b}}$ & 90.8 \\
D $(r$ TpUbc+QuilA repeat) &
\end{tabular}

Reduction rate (\%) was calculated by the following formula: [1- (mean number of cysticerci with vaccination / mean number of cysticerci for control) x 100\%], compared to control group. Different superscript letters indicate the statistically significant differences $(\mathrm{P}<0.05)$ by the non-parametric Mann-Whitney test.

Serological analyses of rabbit sera from samples taken throughout the vaccine study indicate that specific immune responses to the recombinant antigens were produced in the vaccinated animals, with a clear increase in the OD value of $\operatorname{IgG}$ being observed after the 1 st and 2nd immunizations (Figure 3). These levels were significantly higher compared to the levels observed in the QuilA and non-vaccinated groups $(\mathrm{P}<0.01)$. The specific IgG antibody levels in all of the rabbits in the vaccinated groups reached a peak at week 4 after the 1st vaccination $\left(\mathrm{OD}_{450}=1.92\right.$ and 1.88 in groups $\mathrm{C}$ and $\mathrm{D}$, respectively). The IgG levels remained high for the remainder of the experimental period (7 weeks post-challenge). In the QuilA and nonvaccinated groups, the $\mathrm{OD}_{450}$ values of specific IgG remained low throughout the experiment.

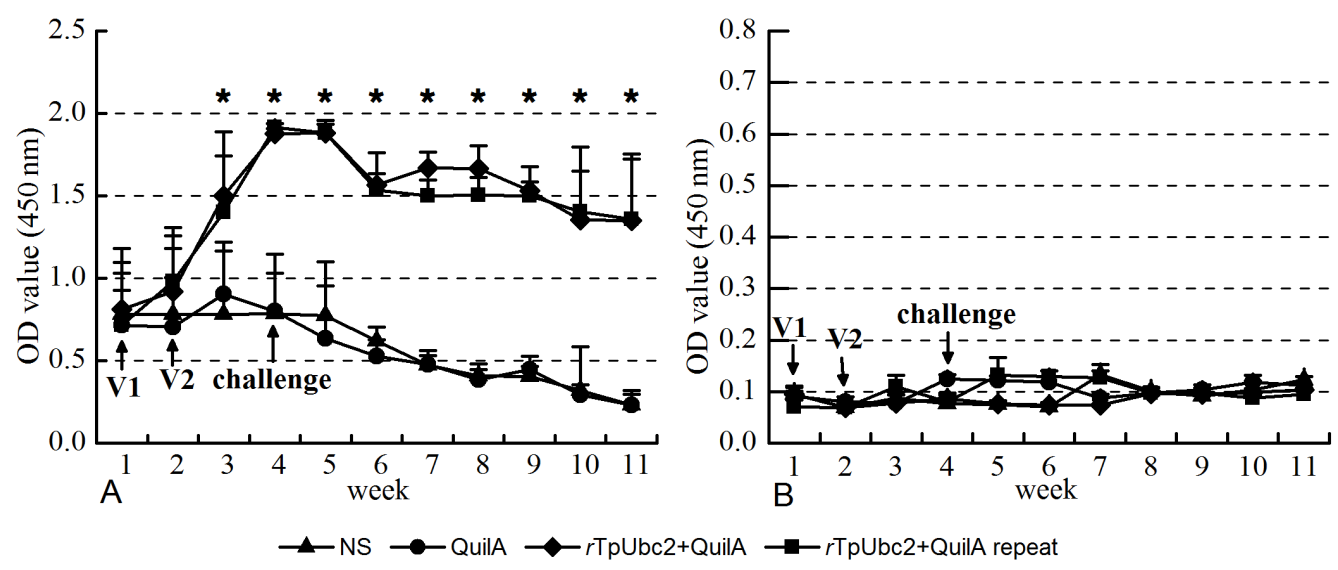

Figure 3. Specific $\operatorname{IgG}(\mathbf{A})$ and $\operatorname{IgA}(\mathbf{B})$ antibody levels in the sera of rabbits immunized with Taenia pisiformis. $r \mathrm{TpUbc} 2$ detected by ELISA. Rabbits were vaccinated twice with $r \mathrm{TpUbc2}$, QuilA, and saline and challenged with 5000 T. pisiformis eggs. The results are reported as means $\pm \mathrm{SD}$. OD values were determined as absorbance at $450 \mathrm{~nm}$. $\mathrm{V} 1$ = first vaccination; $\mathrm{V} 2=$ second vaccination. $* \mathrm{P}<0.01$ compared to NS (control group). 
Due to the lack of an effective secondary anti-rabbit IgA antibody reaction, we chose to measure the levels of total $\operatorname{IgA}$. Compared to the pre-immune serum, the total $\operatorname{IgA}$ serum levels did not change after 2 immunizations in any of the groups. At 4 weeks after challenge, the IgA levels of the vaccinated group, unvaccinated group, and QuilA group remained at a low level $\left(\mathrm{OD}_{450}<0.2\right)$. No statistically significant difference was observed between the $r \mathrm{TpUbc} 2$ group and the control group.

\section{DISCUSSION}

In this study, we described the molecular characterization of TpUbc2 from the $T$. pisiformis oncosphere, and evaluated its ability to induce protection against infection in rabbits. We successfully cloned and expressed the ubiquitin-conjugating enzyme gene TpUbc2, with the TpUbc2 protein showing a high degree of homology to the Asc s 1.0101 allergen of $A$. suum, the Vesv 6.0101 allergen of Vespula vulgaris, and the Sola t 4 allergen of Solanum tuberosum. The prediction of cross-reactivity indicated a highly significant sequence match between TpUbc2 and the A. suum allergens. Thus, TpUbc2 is not a specific allergen for T. pisiformis larvae, and may have a high level of cross-reactivity with the Ubc2 of other nematodes and plants.

Although purified native antigens could theoretically be used in cysticercosis vaccination trials, the lack of an in vitro culture protocol means that it is difficult to obtain large quantities of native material. In previous studies, most researchers have focused on other methods of vaccine production to control cysticercosis, such as recombinant vaccines (Gauci et al., 2012; Jayashi et al., 2012), synthesized peptide vaccines (Huerta et al., 2001; De Aluja et al., 2005; Cruz-Revilla et al., 2006; Gazarian et al., 2012), and phage vaccines (Betancourt et al., 2012). Recently, the efficacy of the oral vs injectable S3Pva-synthetic and S3Pvac-phage vaccines from Taenia solium were compared. The data showed that oral S3Pvac-papaya significantly reduced the expected number of hepatic lesions and peritoneal cysticerci to a similar extent to that of the injectable vaccines (Betancourt et al., 2012). In the current study, the reduction in the recovery of larvae induced by the TpUbc2 recombinant vaccine was 79.3 and $90.8 \%$. These results support the use of $r \mathrm{TpUbc2}$ as a potential candidate to develop a vaccine against T. pisiformis larvae.

Immunization with $r \mathrm{TpUbc} 2$ formulated with QuilA elicited a specific immune response and protection against challenge with $T$. pisiformis eggs, as evidenced by a significant reduction in cysticerci compared to the non-vaccinated control groups. This result indicates that $r \mathrm{TpUbc} 2$ is able to eliminate T. pisiformis larvae through an immunological reaction. The levels of TpUbc2specific serum IgG antibody in vaccinated rabbits, as measured by an ELISA, indicated that rabbits vaccinated with $r \mathrm{TpUbc2}$-QuilA displayed a significantly higher level of specific IgG compared to the control groups. The results also indicated that $r \mathrm{TpUbc} 2$ could be used for the serodiagnosis of T. pisiformis larvae. However, more research is required to substantiate this hypothesis.

The effectiveness of the infection of T. pisiformis eggs in rabbits is variable. Craig and Zumbuehl (1988) found 155 cysts after infection with 1500 parasite eggs. However, in other studies by the same author, only 57 cysts were found after infection with 2000 eggs (Craig, 1984). Another group reported that an average of 8 cysticerci was recovered after challenge with 3300 eggs (Gemmell, 1965; Betancourt et al., 2012). We found an average of 206 cysticerci after infection with 5000 eggs. This variability might be related to the genetic make-up of the rabbits being tested. In addition, the relative infectivity of the particular strains used in the experiments should also be considered. 
The oncosphere has been found to be highly susceptible to attack by the host's immune system (Jabbar et al., 2010). Studies using oncosphere antigens to elicit protective immunity against cysticercosis have been reported by several research groups (Jabbar et al., 2010; Gauci et al., 2012; Jayashi et al., 2012). The conjugation of ubiquitin to a target protein is a cascade enzymatic reaction involving an ubiquitin-activating enzyme (E1), an ubiquitinconjugating enzyme (E2 or UBC), and an ubiquitin-protein ligase (E3). E2 is reacted with activated ubiquitin from E1, and donates ubiquitin monomers or multiubiquitin chains either directly or with the assistance of ubiquitin-protein ligase (E3) to an amino group of a lysine residue. The E2 is an important enzyme that is involved in the ubiquitin-proteasome system at all stages of the cestode lifecycle. In addition, the ubiquitin-proteasome system is also an essential mechanism for protein degradation, cell cycle progression, inflammatory response, transcriptional regulation, and signal transduction in eukaryotes.

The ubiquitin-conjugating (E2) isoenzyme family is characterized by the presence of a highly conserved UBC domain (Hofmann and Pickart, 2001). In humans, 35 active E2 enzymes have been identified to date, while other eukaryotic genomes harbor 16 to $35 \mathrm{E} 2$ family members. The function of $\mathrm{E} 2$ has been extensively studied in yeast, and it appears to have distinct roles in a variety of cell events (Hofmann and Pickart, 2001). Homologous sequences to yeast E2 enzyme genes have also been characterized from other species, e.g., Caenorhabditis elegans (Zhen et al., 1993), Drosophila melanogaster (Koken et al., 1991), and many plants. Song et al. (2004) cloned and expressed the GST-tagged E2 gene of Clonorchis sinensis. The authors also confirmed that this expressed protein was functionally active. To date, the only UBC enzyme genes and proteins reported in Taeniidae studies are limited to bioinformatic analysis and the analysis of preliminary immunoreactivity in Taenia asiatica (Liao et al., 2009). The current study is the first to use a recombinant Ubc2 product as a potential vaccine based on a parasitic antigen.

The immune response elicited against the recombinant $\mathrm{TpUbc} 2$ cestode antigen might be related to the ubiquitin-proteasome protein degradation system; however, further research is required to determine all the factors that are involved.

\section{ACKNOWLEDGMENTS}

Research supported by the Program for Changjiang Scholars and Innovative Research Team in University (PCSIRT) (\#IRT0848). We thank Qin Hu, Cheng Chen, Lan-Ying Wang, $\mathrm{Zu}-\mathrm{Gen}$ Fang, Qing Dong, and Yin-Jiao Luo for technical assistance.

\section{REFERENCES}

Bagrade G, Kirjusina M, Vismanis K and Ozolins J (2009). Helminth parasites of the wolf Canis lupus from Latvia. J. Helminthol. 83: 63-68.

Betancourt MA, De Aluja AS, Sciutto E, Hernandez M, et al. (2012). Effective protection induced by three different versions of the porcine S3Pvac anticysticercosis vaccine against rabbit experimental Taenia pisiformis cysticercosis. Vaccine 30: 2760-2767.

Chen WJ, Niu DS, Zhang XY, Chen ML, et al. (2003). Recombinant 56-kilodalton major outer membrane protein antigen of Orientia tsutsugamushi Shanxi and its antigenicity. Infect. Immun. 71: 4772-4779.

Craig PS (1984). Circulating antigens, antibodies and immune complexes in experimental Taenia pisiformis infections of rabbits. Parasitology 89 (Pt 1): 121-131.

Craig PS and Zumbuehl O (1988). Immunization against experimental rabbit cysticercosis using liposome-associated 
antigen preparations. J. Helminthol. 62: 58-62.

Cruz-Revilla C, Toledo A, Rosas G, Huerta M, et al. (2006). Effective protection against experimental Taenia solium tapeworm infection in hamsters by primo-infection and by vaccination with recombinant or synthetic heterologous antigens. J. Parasitol. 92: 864-867.

De Aluja AS, Villalobos NM, Nava G, Toledo A, et al. (2005). Therapeutic capacity of the synthetic peptide-based vaccine against Taenia solium cysticercosis in pigs. Vaccine 23: 4062-4069.

Finley D, Bartel B and Varshavsky A (1989). The tails of ubiquitin precursors are ribosomal proteins whose fusion to ubiquitin facilitates ribosome biogenesis. Nature 338: 394-401.

Foronda P, Valladares B, Lorenzo-Morales J, Ribas A, et al. (2003). Helminths of the wild rabbit (Oryctolagus cuniculus) in Macaronesia. J. Parasitol. 89: 952-957.

Gauci CG, Jayashi CM, Gonzalez AE, Lackenby J, et al. (2012). Protection of pigs against Taenia solium cysticercosis by immunization with novel recombinant antigens. Vaccine 30: 3824-3828.

Gazarian KG, Solis CF, Gazarian TG, Rowley M, et al. (2012). Synthetic peptide-targeted selection of phage display mimotopes highlights immunogenic features of alpha-helical vs non-helical epitopes of Taenia solium paramyosin: implications for parasite- and host-protective roles of the protein. Peptides 34: 232-241.

Geldhof P, De Maere V, Vercruysse J and Claerebout E (2007). Recombinant expression systems: the obstacle to helminth vaccines? Trends Parasitol. 23: 527-532.

Gemmell MA (1965). Immunological responses of the mammalian host against tapeworm infections. ii. Species specificity of hexacanth embryos in protecting rabbits against Taenia pisiformis. Immunology 8: 270-280.

Hofmann RM and Pickart CM (2001). In vitro assembly and recognition of Lys-63 polyubiquitin chains. J. Biol. Chem. 276: 27936-27943.

Huerta M, De Aluja AS, Fragoso G, Toledo A, et al. (2001). Synthetic peptide vaccine against Taenia solium pig cysticercosis: successful vaccination in a controlled field trial in rural Mexico. Vaccine 20: 262-266.

Jabbar A, Crawford S, Gauci CG, Walduck AK, et al. (2010). Oncospheral penetration glands and secretory blebs are the sources of Taenia ovis vaccine antigens. Infect. Immun. 78: 4363-4373.

Jayashi CM, Kyngdon CT, Gauci CG, Gonzalez AE, et al. (2012). Successful immunization of naturally reared pigs against porcine cysticercosis with a recombinant oncosphere antigen vaccine. Vet. Parasitol. 188: 261-267.

Jia WZ, Yan HB, Guo AJ, Zhu XQ, et al. (2010). Complete mitochondrial genomes of Taenia multiceps, T. hydatigena and T. pisiformis: additional molecular markers for a tapeworm genus of human and animal health significance. $B M C$ Genomics 11: 447.

Koken M, Reynolds P, Bootsma D, Hoeijmakers J, et al. (1991). Dhr6, a Drosophila homolog of the yeast DNA-repair gene RAD6. Proc. Natl. Acad. Sci. U. S. A. 88: 3832-3836.

Kolaskar AS and Tongaonkar PC (1990). A semi-empirical method for prediction of antigenic determinants on protein antigens. FEBS Lett 276: 172-174.

Kyngdon CT, Gauci CG, Rolfe RA, Velasquez Guzman JC, et al. (2006). In vitro oncosphere-killing assays to determine immunity to the larvae of Taenia pisiformis, Taenia ovis, Taenia saginata, and Taenia solium. J. Parasitol. 92: 273281.

Lahmar S, Sarciron ME, Rouiss M and Mensi M (2008). Echinococcus granulosus and other intestinal helminths in semistray dogs in Tunisia: infection and re-infection rates. Tunis Med. 86: 657-664.

Liao XJ, Dai JL, Huang J, Hu XC, et al. (2009). Cloning, expression and immunoassay of ubiquitin-conjugating enzyme E2(UBC E2) gene from Taenia saginata asiatica. J. Xi' an Jiaotong Univ. (Med. Sci.) 30: 395-398.

Lightowlers MW (2006). Vaccines against cysticercosis and hydatidosis: foundations in taeniid cestode immunology. Parasitol. Int. 55 (Suppl): S39-S43.

Martinez-Moreno FJ, Hernandez S, Lopez-Cobos E, Becerra C, et al. (2007). Estimation of canine intestinal parasites in Cordoba (Spain) and their risk to public health. Vet. Parasitol. 143: 7-13.

Rajasekariah GR, Rickard MD and O'Donnell IJ (1985). Taenia pisiformis: protective immunization of rabbits with solubilized oncospheral antigens. Exp. Parasitol. 59: 321-327.

Rashed RM, Whitfield PJ and Lewis JW (1991). The epidemiology of Taenia pisiformis infections in domestic dogs in Cairo. J. Egypt. Soc. Parasitol. 21: 597-610.

Saeed I, Maddox-Hyttel C, Monrad J and Kapel CM (2006). Helminths of red foxes (Vulpes vulpes) in Denmark. Vet. Parasitol. 139: 168-179.

Sambrook J (2001). Molecular Cloning: A Laboratory Manual. Cold Spring Harbor Laboratory, Cold Spring Harbor.

Shield JM, Heath DD and Smyth JD (1973). Light microscope studies of the early development of Taenia pisiformis cysticerci. Int. J. Parasitol. 3: 471-480.

Song L, Chen S, Yu X, Wu Z, et al. (2004). Molecular cloning and characterization of cDNA encoding a ubiquitinconjugating enzyme from Clonorchis sinensis. Parasitol. Res. 94: 227-232.

Genetics and Molecular Research 13 (3): 6148-6159 (2014)

CFUNPEC-RP www.funpecrp.com.br 
Toral-Bastida E, Garza-Rodriguez A, Jimenez-Gonzalez DE, Garcia-Cortes R, et al. (2011). Development of Taenia pisiformis in golden hamster (Mesocricetus auratus). Parasit. Vectors. 4: 147.

Yang D, Fu Y, Wu X and Yue X (2012). Annotation of the transcriptome from Taenia pisiformis and its comparative analysis with three Taeniidae species. PLoS One 7: e32283.

Ye Y and Rape M (2009). Building ubiquitin chains: E2 enzymes at work. Nat. Rev. Mol. Cell Biol. 10: 755-764.

Zhen M, Heinlein R, Jones D, Jentsch S, et al. (1993). The ubc-2 gene of Caenorhabditis elegans encodes a ubiquitinconjugating enzyme involved in selective protein degradation. Mol. Cell Biol. 13: 1371-1377.

Zhou YX, Du AF, Zhang XJ, Wu YM, et al. (2008). Research of harmfulness of Cysticercus pisiformis in rabbit. J. Zhejiang Agricult. Sci. 3: 372-373. 